\title{
Multi-scale characterization of precipitated silica
}

\author{
Manuel Meier $^{\mathrm{a}, *}$, Sebastian Sonnick ${ }^{\mathrm{b}}$, Ermek Asylbekov ${ }^{\mathrm{a}}$, Matthias Rädle ${ }^{\mathrm{b}}$, Hermann Nirschl ${ }^{\mathrm{a}}$ \\ ${ }^{a}$ Karlsruhe Institute of Technology, Institute for Mechanical Process Engineering and Mechanics, Karlsruhe, Germany \\ ${ }^{\mathrm{b}}$ Mannheim University of Applied Sciences, Institute of Process Control and Innovative Energy Conversion, Mannheim, Germany
}

\begin{abstract}
A B S T R A C T
In this study, we performed a multi scale characterization of precipitated silica including small angle X ray scat tering (SAXS), analytical (ultra ) centrifugation as well as electron microscopy and pore size determination tech niques. Information about the primary particles and their size distribution within the aggregate, the aggregate size and its fractal dimension, as well as mesoscopic bulk properties, e.g. porosity and sediment structure analysis, were put into context. The data obtained here allow a statement to be made about the number of primary parti cles per aggregate and the aggregate structure including pore size distribution and pore inlet diameter. The cor relation of the obtained measurement data with mathematical models allows a deeper understanding of the structure and description of fractal aggregates.
\end{abstract}

Keywords:

Precipitated silica

Multi-scale characterization

SAXS

AUC

Porosimetry

\section{Introduction}

Within the last one hundred years, the exchange of goods from countries all over the world has become an important part of the trading business. The longer the way to ship, the more important it is to ensure the cold chain for maintaining product quality. Whereas the fields of ap plication as well as the sizes and shapes of goods are highly variable, the requirements for insulation materials are accordingly clearly defined: high insulation effect at low weight, high transport stability and low production costs. Expanded polystyrene is a material that meets all these requirements. It forms a high porous network with less solid brid ges, resulting in a low thermal conductivity of $<30 \mathrm{~mW} / \mathrm{mK}[1,2]$. In terms of sustainability, oil free materials have been under investigation for their use as insulation material for some time now.

Silica have been an important additive in countless products and ap plications for almost 100 years. The areas of application range from fill ing materials, stabilizers and binders on an industrial scale to special products with a defined size, shape and purity [3 5 ]. The basis for many of these products is the conversion of production from top down to bottom up methods. The most commonly used methods starting from atoms or molecules are flame pyrolysis, emulsion gel and sol gel process [6]. The emulsion gel and the sol gel process are well known processes for the synthesis of spherical colloidal silica

Abbreviations: $d[\mathrm{~nm}]$, Size; $D_{m f}[\quad]$, Fractal dimension of mass; $D_{s f} f$ ], Fractal dimension of surface; $I(q)\left[a . u\right.$.], Scattering intensity; $\left.k_{f} f \quad\right]$, Pre-factor of the fractal dimension; $n, N_{p p}[\quad]$, Number of primary particles per aggregate; $\phi\left[\right.$ ], Porosity; $q\left[n m{ }^{1}\right.$ ] Scattering vector; $\rho\left[\mathrm{gcm}^{3}\right]$, Density; $S_{m}\left[\mathrm{~m}^{2} \mathrm{~g}{ }^{1}\right]$, Specific surface area; $\sigma_{\text {rell }}[\%]$, Relative standard deviation; $z[$ ], Scaling relationship factor.

* Corresponding author.

E-mail address: manuel.meier@kit.edu (M. Meier). nanoparticles with precisely defined particle distributions, e.g. for catal ysis, electronics or pharmaceuticals [7,8]. However, their manufacturing process is quite complex and expensive. The synthesis of fractal silica by flame pyrolysis or precipitation is much cheaper and can be realized on a larger scale. The typical structure of fractal silica is based on primary particles in the size range of a few nanometers which form an aggregate structure up to several micrometers. This loose structure of the aggre gates results in a significantly lower bulk density $\left(\rho_{\text {bulk }} \sim 0.3 \mathrm{gcm}^{-3}\right)$ compared to the density of colloidal silica $\left(\rho_{\mathrm{SiO}_{2}} \sim 2.1 \mathrm{gcm}^{-3}\right)$. The low bulk density and the high contact resistance associated with it makes fractal silica an interesting alternative to conventional insulation mate rials. In addition, these materials have low flammability and good biode gradability, which makes them particularly interesting for building insulation. Insulating panels made of fumed silica have already been tested in many studies (e.g. $[9,10])$ and commercial products are al ready available on market. But the high manufacturing costs limit their scope. Precipitated silica is significantly cheaper to produce. How ever, most of these materials are developed in terms of their effect on the product, but not in terms of particle properties.

Many studies within the last 50 years have faced the challenge of a detailed characterization of fractal aggregate systems, e.g. fumed silica and carbon black (e.g. [11 16]). With growing understanding of the structure of fractal aggregates grew also the desire for a mathematical description of the same. These descriptions in turn form the basis for the mathematical modelling of realistic aggregates and their physical properties [17 21]. A fundamental consideration of the structure of ag gregates based on computer simulations of diffusion limited aggrega tion (DLA) was made by Forrest and Witten [22] and Witten and Sander [23 26] (see Eq. (1)). The equation correlates the number of pri mary particles per aggregate $N_{p p}$ with the primary particle and 
aggregate size $d_{p}$ and $d_{a}$ as well the fractal dimension of the mass $D_{m f}$. The equation also contains a constant pre factor of the fractal dimension $k_{f}$ which is usually determined in combination with the fractal dimen sion of the mass $D_{m f}$. However, the function of the pre factor is de scribed in the literature only vaguely as a scaling factor between the shape of the primary particles and the geometry of the aggregates $[27,28]$. Knowledge of the meaning of the pre factor and its value is, however, of crucial importance in order to enable a realistic representa tion of the aggregates.

$N_{p p} \quad k_{f} \cdot\left(\frac{d_{a}}{d_{p}}\right)^{D_{m f}}$

This work deals with the metrological description of the pre factor of the fractal dimension by a multi scale characterization of precipitated silica. Therefore, information about the particle properties were put into context with mathematical descriptions of fractal aggregates. The metrological description of the aggregate structure included measure ments of the primary particles size and their size distribution within the aggregate, the aggregate size and its fractal dimension, as well as mesoscopic bulk properties, e.g. porosity and sediment structure analysis.

\section{Experimental}

\subsection{Precipitated silica}

The samples GT (Perkasil GT 3000 PD), KS (Perkasil KS 408 PD), CP (CP 513 11202) and MX (Syloid MX109) were purchased from the com pany Grace (USA) and used without further processing.

\subsection{Small angle $X$ ray scattering}

SAXS measurements were performed with an in house developed lab scale USAXS/WAXS device described in our previous works [29 31]. The experimental setup consists of a $1.2 \mathrm{~kW} \mathrm{Cu} \mathrm{K \alpha} \mathrm{X} \mathrm{ray}$ source, a focusing slit shaped $\mathrm{X}$ ray beam realized through a multilayer focusing optical element (Göbel mirror), a block slit collimator system and a Pilatus $100 \mathrm{~K} \mathrm{~S}$ on line detector (co. Dectris Ltd., Switzerland). The detector has a dimension of 83 by $33 \mathrm{~mm}$ with a pixel size of $0.172 \mathrm{~mm}$. The slit shaped $X$ ray beam is recorded along the height of the detector and the scattering is detected along the length. Analyzing scattering data, the intensities of each pixel are integrated over the height of the $\mathrm{X}$ ray beam and then plotted over the detector length. To correct the error of a slit shaped $\mathrm{X}$ ray beam, the background corrected scattering data were desmeared using the direct method according to Singh et al. [32]. The intensity $I(q)$ obtained by SAXS is measured as a function of the scattering vector $q$ with $q=4 \pi / \lambda \cdot \sin (\theta / 2)$. Here, $2 \theta$ is the scattering angle and $\lambda=0.154 \mathrm{~nm}$ the wavelength of the used $\mathrm{Cu}$ $\mathrm{K} \alpha \mathrm{X}$ ray. The probe detector distance is $1240 \mathrm{~mm}$, which corresponds to a smallest resolvable scattering vector $q_{\min }=0.016 \mathrm{~nm}^{-1}$. The scat tering data were recorded for 300 seconds. The primary particle and ag gregate size as well as their fractal dimensions were obtained by the unified fit model $[33,34]$.

\subsection{Analytical (ultra ) centrifugation}

For centrifugation, sample suspensions with $1.5 w t$. \% in deionized water were prepared. To ensure a proper dispersion of the samples, the suspensions were treated under cooling with ultrasonic waves for 10 min (Sonifier 450, co. Branson Ultrasonics, USA). An optical disc centri fuge (AUC) DC24000 (co. CPS Instruments, USA) was used to determine the aggregate size und the size distribution. The average centrifugal ac celeration was $1720 \mathrm{~g}$ at $5850 \mathrm{rpm}$. The density gradient used here consisted of a mixture of a 3 and $5 w t$. \% sucrose solution (Sucrose $\geq 99 \%$, co. Merck, Germany). The sample volume for injection was $100 \mu \mathrm{L}$.
For the determination of the bulk density an optical ultracentrifuge (LUMiFuge, co. LUM, Germany) was used. The suspension $\left(V_{\text {susp }}=450\right.$ $\mu L ; c_{\text {susp }}=1.5 w t . \%$ ) was transferred into a cuvette (width $W=8 \mathrm{~mm}$; depth $D=2 \mathrm{~mm}$; co. LUM, Germany) and centrifuged for $10 \mathrm{~min}$ at $4000 \mathrm{rpm}$ (2020 g). The determination of the sediment height $H$ is per formed by analyzing the transmission profile over the entire cuvette length with a pixel size of $14 \mu \mathrm{m}$. The bulk density is calculated using Eq. (2). The $p H$ value was adjusted by adding $0.1 M$ hydrochloric acid and sodium hydroxide solution (co. Merck, Darmstadt, Germany). Five fold determinations were performed for all samples.

$\rho_{\text {bulk }} \quad \frac{V_{\text {susp }} \cdot C_{\text {susp }}}{W \cdot D \cdot H} \cdot 10^{2}$

\subsection{Electron microscopy}

SEM images were taken with a SEM4500 (co. Hitachi High Technologies Corporation, Tokyo, Japan). The samples were diluted with ethanol (dilution factor $=1: 20$ ) at $20{ }^{\circ} \mathrm{C}$ and treated under cooling with ultrasonic waves for $10 \mathrm{~min}$ to ensure a proper dispersion of the samples. Then, the samples were air dried on a sample holder and coated with a $1.7 \mathrm{~nm}$ layer of platinum paldium.

\subsection{Porosimetry techniques}

The pore size characterization by means of mercury porosimetry was performed using an AutoPore III (co. micromeritics, USA). The deter mination of the surface area and the pore size distribution based on ni trogen ad and desorption according to Brunauer Emmett Teller (BET) and according to Barrett Joyner Halendathe $(B J H)$ were performed using an ASAP 2020 Physisorption (co. micromeritics, USA). To avoid larger air inclusions in the measuring cell due to electrostatic interac tions between the aggregates, the samples were initially pressed at 5 bar. To remove any residual moisture, the samples were heated at 350 ${ }^{\circ} \mathrm{C}$ and 50 mbar for $16 \mathrm{~h}$.

\section{Results and discussions}

To obtain a first inside about the number of the primary particles per aggregate, a scaling relationship factor $z$ (Eq. (3)) can be determined from the primary particle and the aggregate size as well as the fractal di mension of the mass (see Table 1) [14].

$$
z \quad\left(\frac{d_{a}}{d_{p}}\right)^{D_{m f}}
$$

To characterize the primary particle and aggregate properties, small angle $\mathrm{X}$ ray scattering (SAXS) measurements were performed. The analysis included the determination of the primary particle and aggre gate size as well as their fractal dimensions. Fig. 1 shows the sample $G T$ as an example of the evaluation of scattering data using the unified fit model. The evaluation is based on the division of the scattering data into two areas. In the range of small scattering vectors $(q=0.016 \ldots$ $0.1 \mathrm{~nm}^{-1}$ ), the size of the aggregates $d_{a}$ and the fractal dimension of the mass $D_{m f}$ are obtained. In the area of larger scattering vectors

Table 1

Evaluation of the data obtained by SAXS measurements using the unified fit model as well as the scaling relationship factor $\mathrm{z}$ obtained by scattering data

\begin{tabular}{llllll}
\hline Sample name & $D_{s f}[$ & $d_{p}[\mathrm{~nm}]$ & $D_{m f}[$ & $d_{a}[\mathrm{~nm}]$ & $z[\mathrm{]}$ \\
\hline GT & 2.05 & 20.8 & 1.95 & 135.4 & 37.7 \\
KS & 1.88 & 19.8 & 2.07 & 134.6 & 53.3 \\
CP & 1.93 & 11.9 & 1.85 & 163.1 & 126.8 \\
MX & 2.08 & 5.8 & 2.23 & 185.5 & 2267.7 \\
\hline
\end{tabular}




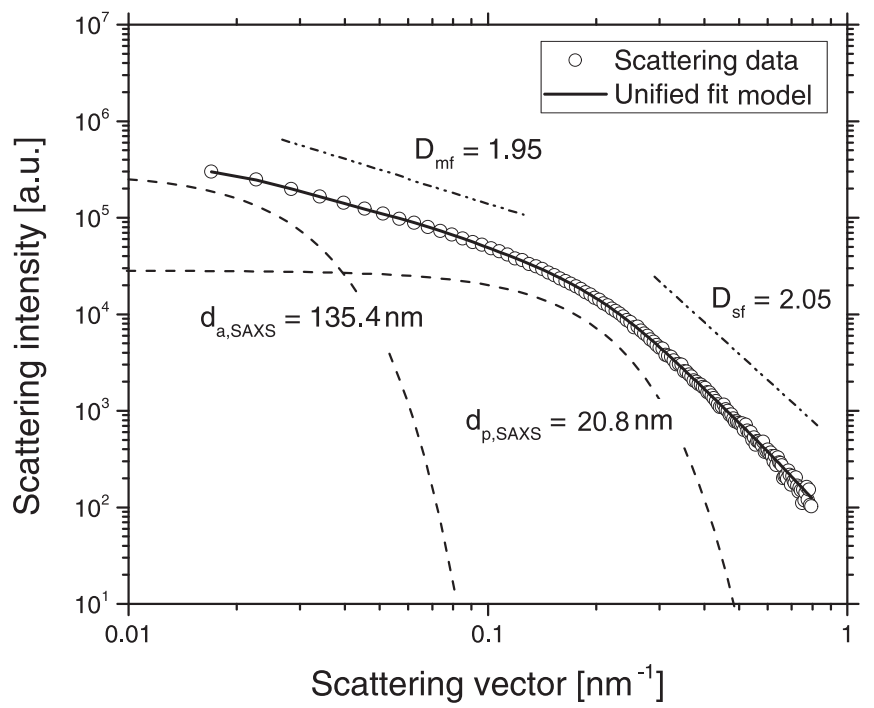

Fig. 1. Scattering data of the sample GT obtained by SAXS. The white circles describe the measured scattering data, the black line the fit from the unified fit model. The dotted lines describe the results of the fit for the different q-ranges: Dashed lines represent the primary particle and aggregate size from the Guinier fits; dashed and dotted lines the fractal dimensions from the power law fits.

$\left(q=0.1 \ldots 0.7 \mathrm{~nm}^{-1}\right)$, the size of the primary particles $d_{p}$ and the fractal dimension of the surface $D_{s f}$ are recorded. An overview over the ob tained results is given in Table 1 . The primary particle size ranges be tween $5.8 \mathrm{~nm}$ for the sample $M X$ up to $20.8 \mathrm{~nm}$ for GT. The aggregate size ranges between $134.6 \mathrm{~nm}$ for $K S$ up to $185.5 \mathrm{~nm}$ for the sample $M X$. The large resolution range of the SAXS system used here enabled a reliable determination of the primary particle size as well as the fractal dimensions. When determining the aggregate size of $M X$, however, it also came close to its resolution limit since a maximum characteristic length of $196 \mathrm{~nm}$ can be resolved with a smallest scattering vector $q_{\min }=0.016 \mathrm{~nm}^{-1}$ (see Bragg equation). Nevertheless, the data and the fits still showed a good agreement here as well. The fractal dimen sion of the surface $D_{s f}$ for all samples are very close to the value 2.0 , in dicating smooth surfaces. The samples GT and KS show a fractal dimension of the mass very close to $2\left(D_{m f, G T}=1.95, D_{m f, K S}=2.07\right)$, in dicating a predominantly disc like $2 \mathrm{D}$ structure. The sample $C P$ seems to have a slightly more elongated structure $\left(D_{m f, C P}=1.85\right)$ whereas $M X$ tends to a more 3D structure $\left(D_{m f, M X}=2.23\right)$ [35]. The scaling rela tionship factor based on scattering data $z$ is given in Table 1. Although the primary particle sizes of the individual particles differ greatly, the sizes of the aggregates are within a narrow range. Therefore, the smaller the primary particle size, the greater is the number of primary particles per aggregate. This is particularly evident for the sample MX. Here, the smallest primary particles form the largest aggregate structure with $>2000$ primary particles per aggregate.

Electron microscopy can provide an analysis of the aggregate struc ture with regard to its shape and in particular the number of primary particles per aggregate. Images of the samples using a scanning electron microscope (SEM) are shown in Fig. 2. The pictures clearly show that it is hardly possible to completely separate the individual aggregates from each other despite previous dispersion with ultrasonic waves. For the samples $G T$ and $K S$ it still works comparatively well. For samples $C P$ and $M X$, however, it is not possible to identify individual aggregates. The high agglomeration tendency of the aggregates effects that already established empirical correlations for the number of primary particles per aggregate from microscopy images cannot be applied here (e.g. $[36,37])$
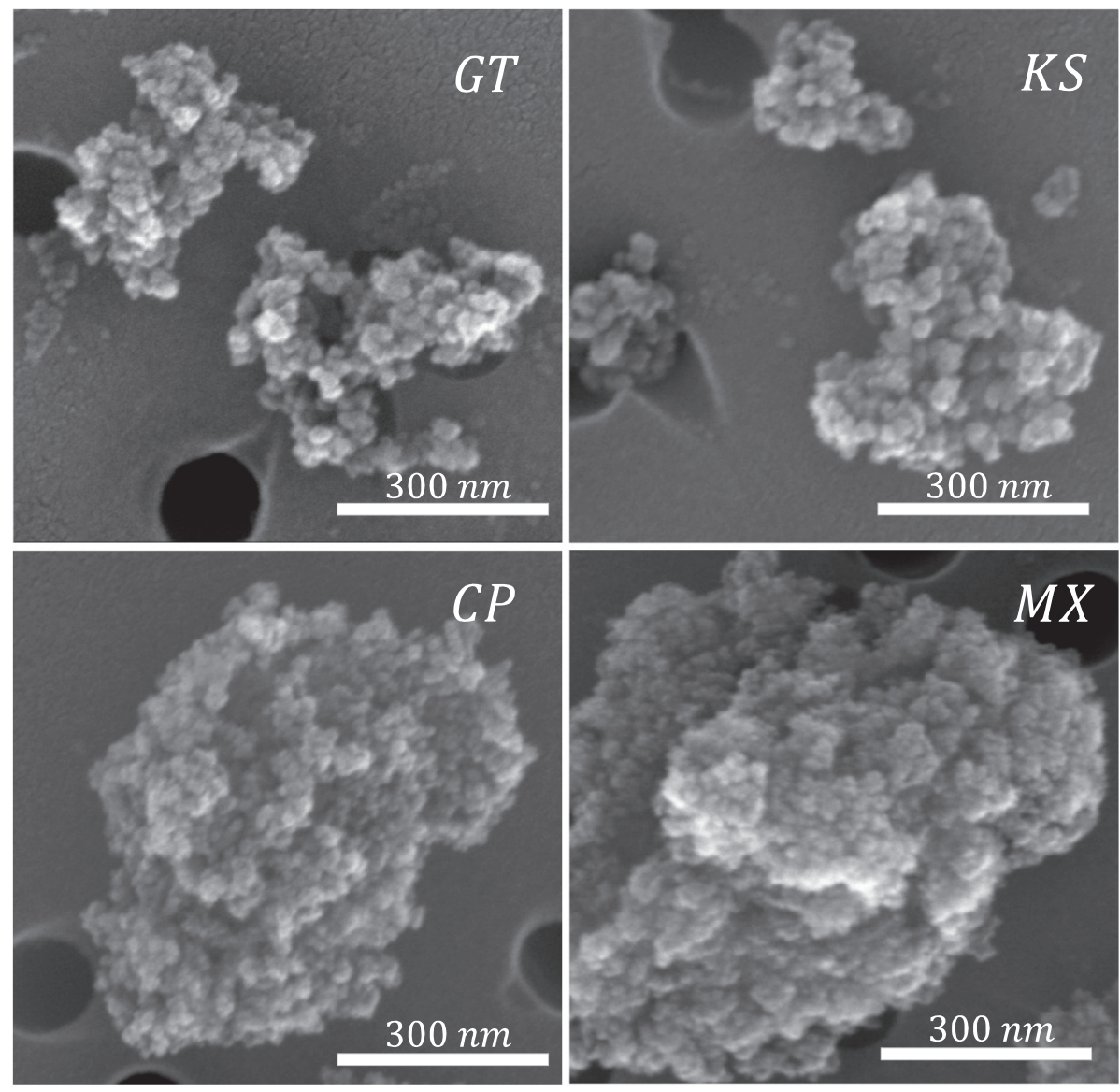

Fig. 2. Images of samples using a scanning electron microscope. 


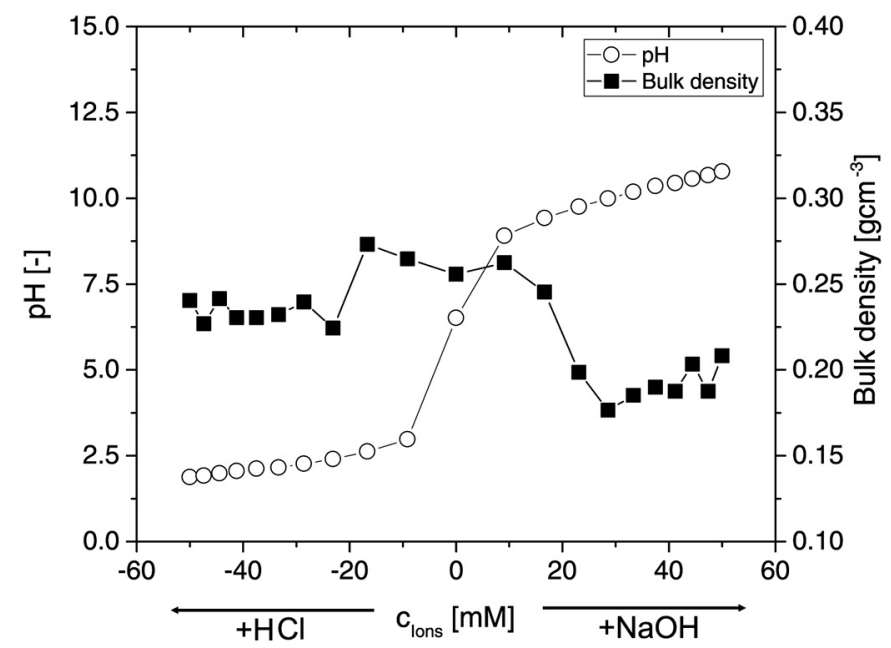

Fig. 3. $p H$ value (white circles) and bulk density (black squares) as a function of the ion concentration for the sample $\mathrm{CP}$ using an optical ultracentrifuge.

Another approach to determine the number of primary particles per aggregate is based on a geometrical consideration. Thus, the volume of the aggregate $V_{a}$ corresponds to the sum of the volumes of the primary particles $V_{p}$ (Eq. (4)).

$V_{a} \sum V_{p}$

This assumption is only useful if the highest possible packing density of the aggregates is assumed. However, this is in contradiction to the model conception of fractal aggregates. If the density of the aggregates and of the primary particles is included in the equation, the error can be corrected. Then, the mass of the aggregate corresponds to the sum of the masses of the primary particles (Eq. (5)).

$m_{a} \sum m_{p}$

The number of primary particles per aggregate corresponds to the ratio of the mass of the aggregate $m_{a}$ to the mass of a primary particle $m_{p}$ (Eq. (6)).

$n \frac{m_{a}}{m_{p}}$

The mass of a primary particle can easily be calculated from its vol ume $V_{p} \quad \frac{\pi}{6} d_{p}^{3}$ and the density of colloidal silica $\left(\rho_{\mathrm{SiO}_{2}} \sim 2.1 \mathrm{gcm}^{-3}\right)$. The mass of an aggregate, on the other hand, is much more difficult to determine. The bulk density as a superior property can be directly deter mined by measurements but the aggregate density is unknown. To de duce the density of the aggregates from the bulk density, it must be ensured that the aggregates occupy their highest packing density. One reason for higher distances between the aggregates is the strong elec trostatic interactions between the aggregates due to the low dead weight. To obtain a maximum value for the bulk density, the repulsive forces between the individual units must be overcome. One approach is based on the application of an external force that presses the aggre gates into a packing that is as dense as possible (compacted bulk density). A disadvantage of this method, however, is that it cannot be ensured whether the highest packing density has been achieved. To en sure this, the pressure can be increased, but this also increases the prob ability that the aggregates will break under the high forces. For this reason, another possibility was sought which, on the one hand, gener ates the highest possible bulk density and, at the same time, largely ex cludes destruction of the aggregates. The method is based on the transferring of the system into an aqueous phase. Resuspension of the precipitated silica in an aqueous environment weakens the repulsive in teractions. The application of a centrifugal acceleration is intended to achieve the highest possible bulk density. This approach bases on the use of an optical ultracentrifuge, which enables the sedimentation of a suspension in the centrifugal field to be monitored in a time resolved manner. Based on the transmission profile at the end of centrifugation, the height of the sediment and thus the bulk density can be determined (see Eq. (2)). In order to avoid any influence of ionic interactions on the results, the $p H$ value was varied by adding positively and negatively charged ions in a range between 1.8 and 10.8. The results of all four sam ples were very close to each other, with only the position and course of the plateau of maximum bulk density per sample varying slightly. For this reason, Fig. 3 shows the example CP for better clarity only. The graph visualizes $\mathrm{pH}$ value (white circles) and the bulk density (black squares) as a function of the ion concentration, indicating a maximum bulk density of $0.265 \mathrm{gcm}^{-3}$ at $\mathrm{pH}$ values between 3 and 10 . The more extreme the $\mathrm{pH}$ values are, the lower is the aggregate density, especially in the alkaline range. The results of all samples in detail are given in Table 2. Since in this $p H$ range the concentration and type of the ions does not seem to have any influence, the mean bulk density was se lected here for further consideration of the aggregate structure (see Eq. (7)).

$\rho_{a} \quad \bar{\rho}_{\text {bulk,pH } 3} 10$

Using the BET method, the specific surface area $S_{m, B E T}$ of the aggre gates can be determined. The specific surface area is of interest in this context as it allows conclusions to be drawn about the size of the pri mary particles. Electron microscopy images show that the primary par ticles are almost perfect spheres. The fractal dimension of the surface $D_{s f}$ from the SAXS analysis suggests that they have an approximately smooth surface (see Table 1 ). This enables the conversion of the specific surface into the corresponding primary particle size $d_{p, B E T}$ (see Eq. (8)). A graphical overview of the obtained specific surface area and the corre spondent primary particle size is shown in Fig. 4 . The higher the specific surface area, the smaller is the correspondent primary particle size. The correspondent primary particle sizes are in a range between $5.5 \mathrm{~nm}$ for the sample MX and $17.7 \mathrm{~nm}$ for the sample $G T$.

$d_{p, B E T} \quad \frac{6}{\rho_{\mathrm{SiO}_{2}} \cdot S_{m, B E T}} \cdot 10^{3}$

To get information not only about the aggregate size, but also about the size distribution, measurements using an optical disc centrifuge (AUC) were performed. The separation principle of the optical disc cen trifuge is based on the migration speed of the aggregates through a su crose gradient in the centrifugal field. The larger the aggregate, the greater is the hydrodynamic diameter and the higher the migration speed of the aggregates. In addition, the sucrose gradient leads to the

Table 2

Evaluation of the data obtained by BET, optical disc centrifuge (AUC) as well as the number of primary particles per aggregate n using the geometrical consideration.

\begin{tabular}{|c|c|c|c|c|c|c|c|c|c|}
\hline Sample name & $S_{m, B E T}\left[m^{2} g{ }^{1}\right]$ & $d_{p, B E T}[n m]$ & $\rho_{a}\left[g_{\mathrm{cm}}{ }^{3}\right]$ & $\rho_{a} / \rho_{\mathrm{SiO} 2}[\mathrm{l}]$ & $d_{a, A U C}[\mathrm{~nm}]$ & $\sigma_{\text {rel }}[\%]$ & $\sigma_{\text {AUC/SAXS }}[\%]$ & $n[$ ] & $z[]$ \\
\hline GT & 161.6 & 17.7 & 0.255 & 0.121 & 120.4 & 13.5 & 7.5 & 38.1 & 37.7 \\
\hline KS & 167.4 & 17.1 & 0.265 & 0.126 & 116.2 & 13.4 & 9.2 & 39.5 & 53.3 \\
\hline $\mathrm{CP}$ & 289.3 & 9.9 & 0.273 & 0.123 & 107.3 & 22.3 & 27.9 & 165.5 & 126.8 \\
\hline MX & 478.6 & 5.5 & 0.252 & 0.120 & 133.2 & 27.5 & 26.2 & 1704.5 & 2267.7 \\
\hline
\end{tabular}




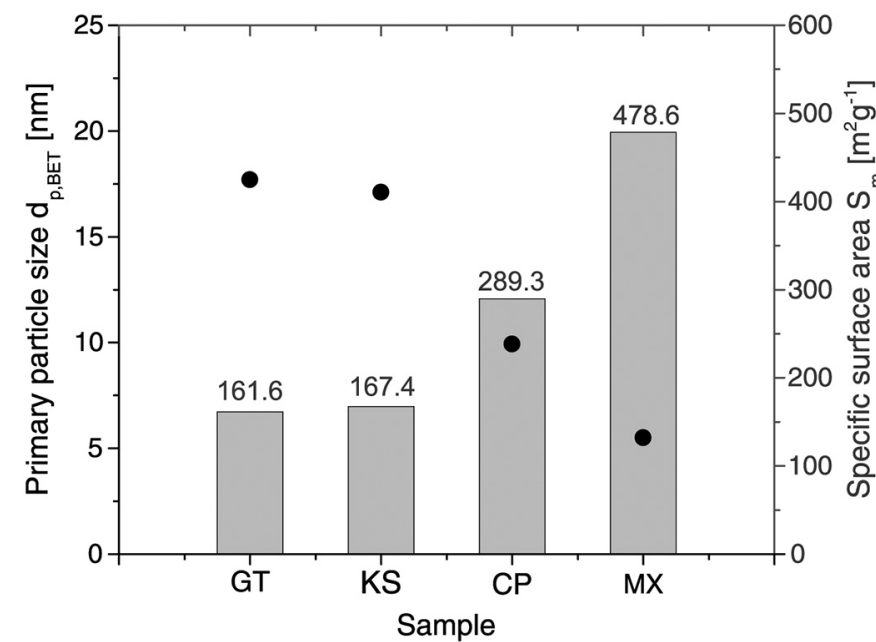

Fig. 4. Specific surface area $S_{m}$ obtained by BET measurements and the correspondent primary particle size $\mathrm{d}_{\mathrm{p}, \mathrm{BET}}$.

separation of aggregates adhering to each other, for example by electro static forces. The density distribution for all samples as well as the stan dard deviation are shown in Fig. 5. The aggregate sizes are in a range between $107.3 \mathrm{~nm}$ for the sample $C P$ and $133.2 \mathrm{~nm}$ for the sample $M X$. The samples with the smallest primary particle sizes have both the smallest and the largest aggregate sizes. Both also show the largest stan dard deviations $\left(\sigma_{\text {rel, } C P}=22.3 \% ; \sigma_{r e l, M X}=27.5 \%\right)$. The samples $G T$ and $K S$ are in the middle in terms of size $\left(d_{a, G T}=120.4 \mathrm{~nm} ; d_{a, K S}=116.2\right.$ $n m)$ but have a significantly lower standard deviation $\left(\sigma_{r e l, G T}=13.5 \%\right.$ $; \sigma_{r e l, K S}=13.4 \%$ ).

The results obtained with BET and $A U C$ are in a good agreement with the results obtained by SAXS. The primary particle sizes from the BET analysis are on average $15 \%$ smaller than those from the SAXS analysis. This is not surprising since particle sizes from SAXS data tend to over weight larger particles and thus shift the mean particle size to larger values. Therefore, the previously made assumptions of spherical pri mary particles with approximately smooth surfaces can be confirmed. In addition, the results indicate that sinter bridges between the primary particles have no significant share in the aggregate structure. The devi ation in aggregate size $\sigma_{A U C / S A X S}$ for the samples GT and $K S$ are $<10 \%$. The samples $C P$ and $M X$, on the other hand, show a much greater deviation of up to $27.9 \%$. An effect that is mentioned before. However, it is notice able that the deviation of the data correlates with the standard deviation

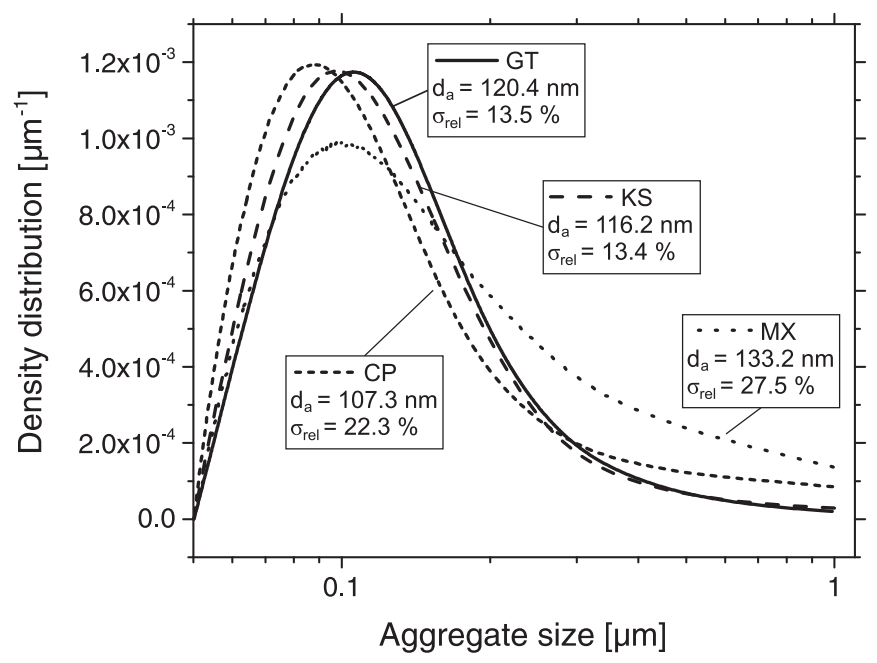

Fig. 5. Density distribution and standard deviation of the aggregate size obtained by optical disc centrifuge (AUC). $\sigma_{\text {rel }}$ of the aggregate size distribution: The higher the standard deviation, the higher is the difference between the aggregate sizes obtained (see Table 2).

Considering a geometrical approach, the obtained aggregate densi ties and primary particle sizes can be used to calculate the number of primary particles per aggregate $n$ (Eq. (9)).

$n \frac{\rho_{a}}{\rho_{\mathrm{SiO}_{2}}} \cdot\left(\frac{d_{a, A U C}}{d_{p, B E T}}\right)^{3}$

In case of spherical primary particles and aggregates, the ratio of the densities of agglomerate to primary particles can be expressed as the compactness of the bulk material, or in other words one minus the po rosity $\phi$. If the density of the aggregates corresponds to that of the pri mary particles, this ratio becomes one, analogous to the behavior of a solid material. The lower the value of the ratio, the looser the bulk seems to be packed. If these aspects are summarized, the number of pri mary particles per aggregate is obtained from a geometric consideration of the primary particles and the aggregates as well as the porosity of the aggregates (see Eq. (10)).

$n \quad(1-\phi) \cdot\left(\frac{d_{a, A U C}}{d_{p, B E T}}\right)^{3}$

Comparing these results with the scaling relationship factor $z$, it can be observed that the orders of magnitude for all samples agree very well (see Table 2). Smaller deviations can occur because the exponent plays a decisive role in determining the number of particles per aggregate, es pecially when calculating the scaling relationship factor. In conclusion, it can be stated that both approaches, the analysis of the scattering prop erties and the structural properties, lead to very similar results.

The consideration of the structure of aggregates based on computer simulations of diffusion limited aggregation (Eq. (1)) shows clearly similarities to the equations for the scaling relationship factor $z$ (Eq. (3)) and the number of primary particles per aggregate $n$ using the geometric consideration (Eq. (9)). In comparison to the scaling rela tionship factor, the pre factor of the fractal dimension $k_{f}$ is set to one. In the geometric consideration of the aggregate structure, this pre factor corresponds to the compactness of the aggregates $(1-\phi)$. The geomet ric consideration is supported by the fact that the aggregate structure is also taken into account due to its compactness. But the further the frac tal dimension of mass deviates from that of a sphere $\left(D_{m f, \text { sphere }}=3\right)$, the greater is the deviation. Thus, a consideration of the results exclusively from scattering or geometric consideration does not result in a further gain in knowledge. Consequently only the possibility remains to con nect both considerations with each other. Since the sizes determined for the primary particles and aggregates were within a narrow value range for all measurement techniques, they can be used as required. When Eq. (3) is set equal to Eq. (9), the pre factor of the fractal dimen sion $k_{f}$ solves to (Eq. (11)):

$k_{f} \quad(1-\phi)\left(\frac{d_{a}}{d_{p}}\right)^{3} D_{m f}$

The equation makes it possible to determine the pre factor sepa rately from the fractal dimension and of imaging techniques. The values obtained for $k_{f}$ range from 0.75 to $2.64\left(k_{f, G T}=0.86 ; k_{f, K S}=0.75 ; k_{f, C P}\right.$ $\left.=2.64 ; k_{f, M X}=1.73\right)$. How the actual value of the pre factor is to be assessed, however, could not be conclusively clarified here either. In particular, since the ratio of the primary particles and aggregate sizes are included in the calculation, the pre factors behave very differently for the individual samples. However, the results correspond well to the results of other studies (e.g. [38,39]).

To proof the statements made on the porosity of the aggregates and to obtain further information about the pore structure of the materials, 


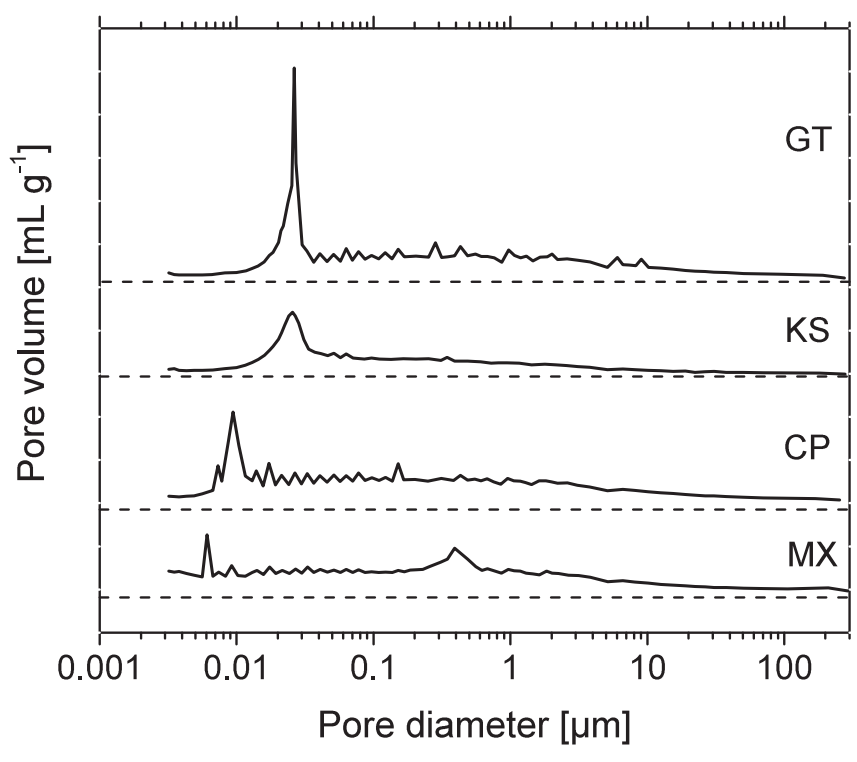

Fig. 6. Results of the mercury porosimetry. The dashed lines represent the abscissa of each graph.

mercury porosimetry was used to analyze meso and macropores as well as the adsorption and desorption of nitrogen to characterize nanoscopic porosity ( $\mathrm{BJH}$ method). In order to avoid larger air inclusions within the measuring cell due to electrostatic interactions between the aggregates, the samples were initially pressed at $5 \mathrm{bar}$. The results of the mercury porosimetry in a range between 0.003 and 50 microns are given in Fig. 6 . The graphs show the pore volume dependent on the pore size per gram sample. For a better comparability of the results the curves were arranged on top of each other. The dashed lines repre sent the abscissa of each graph. All samples show a wide pore size dis tribution and more or less the same course for larger pore sizes. For smaller pore sizes, significant differences in the pore volume and the as sociated pore size can be observed (see Table 3 ). The larger the pore size, the larger is the associated pore volume. It is also noticeable that the larger the primary particle size, the larger is the pore size. In addi tion, the $M X$ sample shows a further peak at larger pore sizes ( $d_{\text {pore }}=$ $355 \mathrm{~nm}$ ). However, it could not be conclusively clarified here whether this is a consequence of the loading of the sample with high pressures, or whether it is actually the case.

The porosity obtained by mercury porosimetry $\phi_{\mathrm{Hg}}$ behaves analo gously to the pore volume for the associated pore sizes: the higher the pore volume, the greater is the porosity. Comparing the results of the porosities by means of mercury porosimetry with the values obtained for the pre factor of the fractal dimension $k_{f}$ for a fractal dimension of 3 , these correspond very well with each other. This result reinforces the assumption that the pre factor can be seen as a benchmark of the compactness of the aggregates, and thus of their porosity. At the same time, this result shows that the porosity of the bulk material is almost exclusively influenced by the porosity of the individual aggregates. Mer cury porosimetry has the advantage of covering a wide range of pore sizes in the meso and macroscopic range. However, if the pores are too small, a correspondingly high pressure must be applied to fill the pores. With less stable samples, this can lead to a change in the

Table 3

Results obtained by $\mathrm{Hg}$ and nitrogen porosimetry.

\begin{tabular}{llllll}
\hline Sample name & $d_{\text {pore } \mathrm{Hg}}[\mathrm{nm}]$ & $\phi_{\mathrm{Hg}}[\%]$ & 1 & $\phi[$ & $d_{\text {pore, BJH }}[\mathrm{nm}]$ \\
\hline GT & 28.3 & 89.7 & 0.124 & 43.6 \\
KS & 25.2 & 86.6 & 0.126 & 40.4 \\
CP & 9.1 & 87.4 & 0.130 & 33.1 \\
MX & 5.8 & 85.7 & 0.120 & 14.0 \\
\hline
\end{tabular}

pore structure or even to collapse. Therefore, besides measurements with mercury porosimetry, measurements with nitrogen were carried out. Next to measurements using the BET method, the BJH method can be used for the determination of the size distribution of nanoscale pores. The results of the $B J H$ method show the same tendency as the re sults obtained by the mercury porosimetry: the larger the size of the pri mary particles, the larger is the pore size. In general, the pore sizes from the $\mathrm{BJH}$ method are larger than those of the mercury porosimetry. How ever, this is not surprising, since the mercury porosimetry determines the pore inlet diameter associated with the pore. This must be smaller than the enclosed pore determined by the BJH method. The deviation of both pore diameters from each other, however, allows conclusions to be drawn about the pore structure itself. The inlet pore diameters of the GT and KS samples are approximately $35 \%$ smaller than the pores themselves. This means that the samples do not contain large enclosed pore structures. The samples $C P$ and $M X$ deviate from this behavior. There, the pore diameters deviate by up to $72 \%$, which in turn suggests large pore inclusions in the structures. It is noticeable that aggregates of small primary particles tend to form enclosed porous structures.

\section{Conclusion}

In this study, we performed a multi scale characterization of four precipitated silica including small angle $X$ ray scattering (SAXS), ana lytical (ultra ) centrifugation as well electron microscopy and pore size characterization techniques. Information about the primary particle and aggregate size as well as their fractal dimension of surface and mass were obtained by SAXS measurements. The scattering data showed pri mary particle sizes in a range between 5.8 and $20.8 \mathrm{~nm}$ with smooth sur faces. The fractal dimension of the mass varied between 1.85 and 2.25 indicating a predominately disc like structure of the aggregates. The mean aggregate sizes obtained by optical disc centrifugation range be tween 107.3 and $133.2 \mathrm{~nm}$ and are in a good agreement with results ob tained by SAXS. The use of an optical ultracentrifuge showed an aggregate density of approximately $0.26 \mathrm{gcm}^{-3}$ and enabled the calcu lation of the number of primary particles per aggregate in a geometrical consideration. The comparison of the mathematical description of frac tal aggregates with measurements of the pore size distribution by means of mercury porosimetry reinforces the assumption that the pre factor of the fractal dimension $k_{f}$ can be seen as a benchmark of the compactness of the aggregates, and thus of the sediment porosity.

\section{Acknowledgements}

The research leading to these results has received funding from the German Federation of Industrial Research Associations(ZF 4005108KI7).

\section{References}

[1] L.I.C. Sandberg, T. Gao, B.P. Jelle, A. Gustavsen, Synthesis of hollow silica nanospheres by sacrificial polystyrene templates for thermal insulation applications, Adv. Mater. Sci. Eng. 2013 (2013).

[2] R. Caps, J. Fricke, Thermal conductivity of opacified powder filler materials for vacuum insulations, Int. J. Thermophys. 21 (2000) 445-452.

[3] D.L. Green, J.S. Lin, Y.-F. Lam, M.Z.C. Hu, D.W. Schaefer, M.T. Harris, Size, volume fraction, and nucleation of Stober silica nanoparticles, J. Colloid Interface Sci. 266 (2003) 346-358.

[4] R.K. Iler, The colloid chemistry of silica and silicates, Soil Sci. 80 (1955) 86

[5] C.J. Brinker, G.W. Scherer, Sol $\rightarrow$ gel $\rightarrow$ glass: I. gelation and gel structure, J. NonCryst. Solids 70 (1985) 301-322.

[6] I.A. Rahman, V. Padavettan, Synthesis of silica nanoparticles by sol-gel: sizedependent properties, surface modification, and applications in silica-polymer nanocomposites; a review, J. Nanomater. 2012 (2012) 8.

[7] S. Fouilloux, A. Désert, O. Taché, O. Spalla, J. Daillant, A. Thill, SAXS exploration of the synthesis of ultra monodisperse silica nanoparticles and quantitative nucleation growth modeling, J. Colloid Interface Sci. 346 (2010) 79-86.

[8] J.S. Ritva Lindberg, Göran Sundholm, Preparation of silica particles utilizing the solgel and the emulsion-gel processes, Colloids Surf. A Physicochem. Eng. Asp. 99 (1995) 79-88. 
[9] H. Schwab, U. Heinemann, A. Beck, H.-P. Ebert, J. Fricke, Dependence of thermal conductivity on water content in vacuum insulation panels with fumed silica kernels, J. Therm. Envel. Build. Sci. 28 (2005) 319-326.

[10] J. Fricke, U. Heinemann, H.P. Ebert, Vacuum insulation panels-from research to market, Vacuum 82 (2008) 680-690.

[11] D.K. Rai, G. Beaucage, K. Vogtt, J. Ilavsky, H.K. Kammler, In situ study of aggregate topology during growth of pyrolytic silica, J. Aerosol Sci. 118 (2018) 34-44.

[12] A. Mulderig, G. Beaucage, K. Vogtt, H. Jiang, V. Kuppa, Quantification of branching in fumed silica, J. Aerosol Sci. 109 (2017) 28-37.

[13] R. Ramachandran, G. Beaucage, A.S. Kulkarni, D. McFaddin, J. Merrick-Mack, V. Galiatsatos, Branch content of metallocene polyethylene, Macromolecules 42 (2009) 4746-4750.

[14] G. Beaucage, Determination of branch fraction and minimum dimension of massfractal aggregates, Phys. Rev. E 70 (2004), 031401.

[15] A.V. Filippov, M. Zurita, D.E. Rosner, Fractal-like aggregates: relation between morphology and physical properties, J. Colloid Interface Sci. 229 (2000) 261-273.

[16] M.L. Eggersdorfer, D. Kadau, H.J. Herrmann, S.E. Pratsinis, Aggregate morphology evolution by sintering: number and diameter of primary particles, J. Aerosol Sci. 46 (2012) 7-19.

[17] E. Guesnet, R. Dendievel, D. Jauffrès, C.L. Martin, B. Yrieix, A growth model for the generation of particle aggregates with tunable fractal dimension, Physica A 513 (2019) 63-73.

[18] C. Schilde, C.F. Burmeister, A. Kwade, Measurement and simulation of micromechanical properties of nanostructured aggregates via nanoindentation and DEM-simulation, Powder Technol. 259 (2014) 1-13.

[19] R. Ceolato, M.J. Berg, N. Riviere, Spectral and angular light-scattering from silica fractal aggregates, J. Quant. Spectrosc. Radiat. Transf. 131 (2013) 160-165.

[20] J. Oberdisse, P. Hine, W. Pyckhout-Hintzen, Structure of interacting aggregates of silica nanoparticles in a polymer matrix: small-angle scattering and reverse Monte Carlo simulations, Soft Matter 3 (2007) 476-485.

[21] M. Grzegorczyk, M. Rybaczuk, K. Maruszewski, Ballistic aggregation: an alternative approach to modeling of silica sol-gel structures, Chaos, Solitons Fractals 19 (2004) 1003-1011.

[22] S.R. Forrest, J.T.A. Witten, Long-range correlations in smoke-particle aggregates, J. Phys. A Math. Gen. 12 (1979) L109.

[23] T.A. Witten, L.M. Sander, Diffusion-limited aggregation, Phys. Rev. B 27 (1983) 5686-5697.
[24] T.A. Witten, L.M. Sander, Diffusion-limited aggregation, a kinetic critical phenomenon, Phys. Rev. Lett. 47 (1981) 1400-1403.

[25] L.M. Sander, Z. Cheng, R. Richter, Diffusion-limited aggregation in three dimensions, Phys. Rev. B 28 (1983) 6394.

[26] L.M. Sander, Diffusion-limited aggregation: a kinetic critical phenomenon? Contemp. Phys. 41 (2000) 203-218.

[27] E. Goudeli, M.L. Eggersdorfer, S.E. Pratsinis, Coagulation of agglomerates consisting of polydisperse primary particles, Langmuir 32 (2016) 9276-9285.

[28] W.R. Heinson, C.M. Sorensen, A. Chakrabarti, A three parameter description of the structure of diffusion limited cluster fractal aggregates, J. Colloid Interface Sci. 375 (2012) 65-69.

[29] M. Meier, J. Ungerer, M. Klinge, H. Nirschl, Synthesis of nanometric silica particles via a modified Stöber synthesis route, Colloids Surf. A Physicochem. Eng. Asp. 538 (2018) 559-564.

[30] M. Meier, J. Ungerer, M. Klinge, H. Nirschl, Formation of porous silica nanoparticles at higher reaction kinetics, Powder Technol. 339 (2018) 801-808.

[31] A. Gutsche, M. Meier, X. Guo, J. Ungerer, H. Nirschl, Modification of a SAXS camera to study structures on multiple scales, J. Nanopart. Res. 19 (2017) 321.

[32] M.A. Singh, S.S. Ghosh, R.F. Shannon, A direct method of beam-height correction in small-angle X-ray scattering, J. Appl. Crystallogr. 26 (1993) 787-794.

[33] G. Beaucage, Approximations leading to a unified exponential/power-law approach to small-angle scattering, J. Appl. Crystallogr. 28 (1995) 717-728.

[34] G. Beaucage, Small-angle scattering from polymeric mass fractals of arbitrary massfractal dimension, J. Appl. Crystallogr. 29 (1996) 134-146.

[35] D.W. Schaefer, J.E. Martin, P. Wiltzius, D.S. Cannell, Fractal geometry of colloidal aggregates, Phys. Rev. Lett. 52 (1984) 2371-2374.

[36] Ü.Ö. Köylü, G.M. Faeth, T.L. Farias, M.G. Carvalho, Fractal and projected structure properties of soot aggregates, Combust. Flame 100 (1995) 621-633.

[37] D.W. Schaefer, A.J. Hurd, Growth and structure of combustion aerosols: fumed silica, Aerosol Sci. Technol. 12 (1990) 876-890.

[38] F.X. Ouf, J. Yon, P. Ausset, A. Coppalle, M. Maillé, Influence of sampling and storage protocol on fractal morphology of soot studied by transmission electron microscopy, Aerosol Sci. Technol. 44 (2010) 1005-1017.

[39] Ü.Ö. Köylü, C.S. McEnally, D.E. Rosner, L.D. Pfefferle, Simultaneous measurements of soot volume fraction and particle size/microstructure in flames using a thermophoretic sampling technique, Combust. Flame 110 (1997) 494-507. 
Karlsruher Institut für Technologie

\section{Repository KITopen}

Dies ist ein Postprint/begutachtetes Manuskript.

Empfohlene Zitierung:

Meier, M.; Sonnick, S.; Asylbekov, E.; Rädle, M.; Nirschl, H.

Multi-scale characterization of precipitated silica.

2019. Powder technology, 354.

doi: 10.5445/IR/1000096145

Zitierung der Originalveröffentlichung:

Meier, M.; Sonnick, S.; Asylbekov, E.; Rädle, M.; Nirschl, H.

Multi-scale characterization of precipitated silica.

2019. Powder technology, 354, 45-51.

doi: $10.1016 /$ j.powtec.2019.05.072

Lizenzinformationen: CC BY-NC-ND 4.0 